\title{
Oesophageal Variceal Band Ligation using a Saeed Six-Shooter Multiband Ligator; Experience at Jos University Teaching Hospital, Nigeria: case report
}

\author{
*Ladep N G, **Sule J, *Umar S M, *Obienu O, *Anyanechi C, *Okeke E N \\ *Department of Medicine, Jos University Teaching Hospital **ECWA Evangel Hospital, Jos, Plateau state, Nigeria
}

\begin{abstract}
Background: Oesophageal varices are common sequelae of cirrhosis, which when they bleed can be difficult to control. Oesophageal variceal band ligation, being the best modality for controlling variceal bleeding has not been common practice in West Africa, a region recognized to have a high prevalence of liver diseases. We present a case of band ligation of the varices of a patient that presented to our hospital with variceal haemorrhage, secondary to liver cirrhosis.

Method: We started by presenting a summary of the presentation of a 31 year old man at the Jos University Teaching Hospital and how we established the diagnosis of grade IV bleeding oesophageal varices. The subject had four bands applied to the oesophageal varices using a saeed six shooter oesophageal Multi-band ligator (North Carolina, USA) using a forward- viewing GIF P30 gastroscope (Tokyo, Japan) with the bands mounted on an Opti-vu barrel- shaped piece. He had a total of three sessions after which he was maintained on propranolol.

Result: The last recheck endoscopy demonstrated obliterated varices after which he was maintained on propranolol.
\end{abstract}

Conclusion: We present a case of successful variceal band ligation of a cirrhotic with extensive oesophageal varices presenting in a resource- constraint medical setting.

\section{Date accepted for publication: $10^{\text {th }}$ October 2007 \\ Nig J Med 2008; 110 - 111 \\ Copyright(2008 Nigerian Journal of Medicine}

\section{CASE PRESENTATION}

Z.A. is a 31-year-old civil servant from Central Nigeria who had presented at the Medical Emergency Unit of the Jos University Teaching Hospital with a 2-week history of having vomited different amounts of fresh blood intermittently. The vomited blood was bright red and presented as clots, which were estimated to be about 3 litres total volume within the first 3 days. There were associated melaena, dizziness, headache, but there was no significant change in urine output. He had no abdominal pain, but had had abdominal swelling and was noted to have obviously icteric sclerae 4 weeks prior to the presenting episode of haematemesis. On previous presentations he had received a total of 15 units of whole blood within a 7-month period.

He had not been confirmed to be a case of sickle cell anaemia and had not taken any alcohol. There was no history of multiple sexual partners.

Examination revealed a young, chronically ill man, who was pale and jaundiced. He had leuconychia, pigmented palms, bilateral gynaecomastia, sparse axillary hair and testicular atrophy. Examination revealed a distended abdomen having no visible distended superficial veins. He had a reduced liver span of $6 \mathrm{~cm}$; an enlarged spleen of $11 \mathrm{~cm}$ and ascites was demonstrable by fluid thrill. The pulse rate was 92 beats/min and the systemic blood pressure was 90/50 $\mathrm{mmHg}$.

His serum bilirubin, alanine and aspartate transaminases were marginally elevated of $20 \mu \mathrm{m} / \mathrm{l}$, $50 \mathrm{U} / \mathrm{l}$ and $42 \mathrm{U} / \mathrm{l}$ respectively, while the total serum proteins $(52 \mathrm{~g} / \mathrm{l})$ as well as albumin $(25 \mathrm{~g} / \mathrm{l})$ were low. He was hepatitis $\mathrm{C}$ antibody positive, but was non reactive to HBsAg and HIV. An ultrasound examination of the abdomen revealed a shrunken liver with coarsened architecture; the spleen was confirmed to be enlarged and there was ascites.

He was resuscitated; including administration of intravenous fluids and subsequently transfused with 4 units of whole blood. He had an upper gastrointestinal endoscopy done to determine the source of bleeding: the findings of which were grade IV oesophageal varices with red whale signs, blood clots and actively bleeding. There was also evidence of congestive gastropathy. The duodenum was normal.

He had four bands applied to the oesophageal varices using a Saeed Six shooter oesophageal Multi-band Ligator (North Carolina, USA) using a forward-viewing GIF P30 gastroscope (Tokyo, Japan) with the bands mounted on an Opti-vu barrel-shaped piece (figure 1). A recheck gastroscopy 2 weeks later revealed that the varices were less in number. These were rebanded. Two weeks later, only one varix could be visualized (figures 2) which was further banded. A recheck gastroscopy revealed absent varices and he had been on propranolol $80 \mathrm{mg}$ bid since then. 


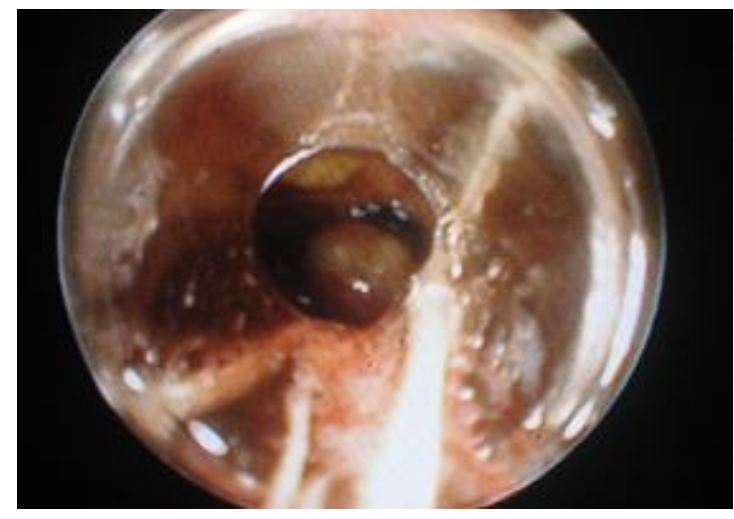

Figure 1: Actively bleeding oesophageal varix with a band applied at its base after suction into the Opti-vu barrelshaped cup at the tip of the gastroscope.

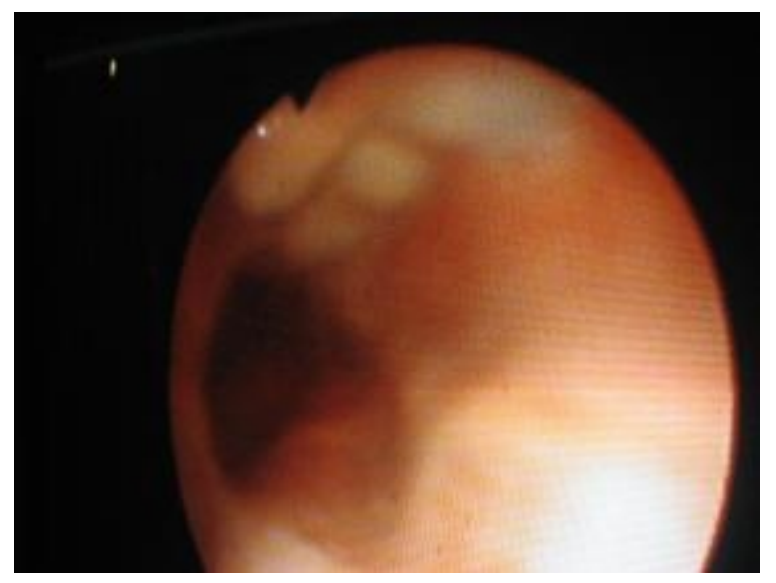

Figure 2: Solitary varix shown here after two sessions of banding

\section{DISCUSSION}

Oesophageal varices are abnormally distended veins in the oesophageal mucosa due to portal hypertension. This results from increased resistance to the blood flow into the liver. Owing to the location of these varices in the lower oesophagus and/or the upper portion of the stomach, bleeding from them is often difficult to control. Pharmacological therapy, including the use of propranolol, balloon tamponade, endoscopic sclerotherapy, band ligation, transjugular intrahepatic

\section{REFERENCES}

1. Van Stiegmann G, Cambre T, Sun JH. A new endoscopic elastic band ligating device. Gastrointest Endosc. 1986; 32:230-233.

2. Stiegmann GV, Goff JS, Sun JH, Davis D, Silas D. Technique and early clinical results of endoscopic variceal ligation (EVL) Surg Endosc 1989; 3:73-78

3. Olubuyide IO. Natural history of liver cirrhosis in 116 Nigerians. EastAfrMed J. 1996; 73:233-235.

4. Achinge $G$. Prevalence of oesophageal varices among patients with liver cirrhosis in Jos. National Postgraduate Medical College dissertation. May 2005:1-89 portocaval shunt surgery and portal-systemic shunt surgery are treatment options.

Two endoscopic procedures available for bleeding varices include sclerotherapy and band ligation. Sclerotherapy was first introduced in 1939 using a rigid oesophagoscope while endoscopic variceal band ligation has evolved to be the preferred first line modality for the endoscopic treatment of oesophageal variceal bleeding. This was introduced in $1989^{1}$ and has then been used for years in the treatment of haemorrhoids ${ }^{2}$. Newer banding devices allow multiple bands to be deployed with the endoscope in place obviating the potential complications of oesophageal perforation. The management of variceal bleeding in our subregion has been, at best, conservative. This has resulted in several preventable deaths. For example, in one report from Nigeria, it was shown that of the causes of mortality among cirrhotics, $40 \%$ was attributable to variceal haemorrhage ${ }^{3}$.

Band ligation of varices is relatively easy to learn and multiple studies have demonstrated that it has a remarkably low complication rate. The need for this procedure cannot be overemphasized as approximately $75 \%$ of cirrhotics attending gastroscopic examinations have been demonstrated to have varices ${ }^{4}$ at our centre underscoring the need to give prospective bleeders some prophylaxis ${ }^{5}$. There are a few reports of oesophageal band ligation from Africa ${ }^{6}$ and none from the West African Subregion. This procedure has been shown to be both beneficial for actively bleeding varices as well as prevention of future bleed. ${ }^{7,8}$

In conclusion, we present a case of successful variceal band ligation of a cirrhotic with extensive oesophageal varices presenting in a resource-constraint medical setting. We suggest that it would be very helpful if endoscopists in the West African subregion acquired this skill so as to abate the unknown havoc bleeding oesophageal varices is wreaking in our population.

5. Sarin SK, Wadhawan M, Agarwal SR, Tyagi P, Sharma BC. Endoscopic variceal ligation plus propranolol versus endoscopic variceal ligation alone in primary prophylaxis of variceal bleeding. Am J Gastroenterol 2005; 100:805-807

6. Jani PG. Endoscopic variceal band ligation; a local experience. East African Medical Journal 2004; 81: 212-214

7. Leszczyszyn J ; Jebski I ; Massopust R ; Skoczylas M ; Janus W. Results of rubber band ligation of esophageal varices. Med SciMonit. 2001; 7 Suppl 1:287-91

8. Khuroo MS, Khuroo NS, Farahat KLC, Khuroo YS, Sofi AA, Dahab ST. Meta-Analysis: Endoscopic Variceal Ligation for Primary Prophylaxis of Oesophageal Variceal Bleeding. Aliment Pharmacol Ther. 2005;21:347-361. 